\title{
Symétrie des solutions d'équations semi-linéaires elliptiques
}

\author{
Jean DOLBEAULT ${ }^{a}$, Patricio FELMER ${ }^{\text {b }}$
}

a Ceremade (UMR CNRS no. 7534), Université Paris IX-Dauphine, Place de Lattre de Tassigny, 75775 Paris Cédex 16, France.

Courriel : dolbeaul@ceremade.dauphine.fr

b DIM, Universidad de Chile, Casilla 170 Correo 3, Santiago, Chili.

Courriel : pfelmer@dim.uchile.cl

\begin{abstract}
Résumé. Dans cette note, nous présentons des résultats de symétrie et de monotonie correspondant à deux cas où le théorème classique de B. Gidas, W.-M. Ni et L. Nirenberg ne s'applique pas. Lorsque l'équation est non-autonome, nous introduisons une comparaison avec un problème linéaire. Lorsque la nonlinéarité n'est pas Lipschitz, nous utilisons des méthodes locales d'hyperplans mobiles, d'inversion locale et de continuation unique. La symétrie usuelle est alors remplacée par une notion de symétrie radiale locale.
\end{abstract}

\section{Symmetry of the solutions of semilinear elliptic equations}

\begin{abstract}
In this note, we present symmetry and monotonicity results corresponding to two cases where the classical theorem by B. Gidas, W.-M. Ni and L. Nirenberg does not apply. When the equation is non-autonomous, we introduce a comparison with a linear problem. When the nonlinearity is non Lipschitz, we use local moving hyperplanes, local inversion and unique continuation methods. The usual notion of symmetry is then replaced by a notion of local radial symmetry.
\end{abstract}

\section{Abridged English Version}

Consider the unit ball $B=B(0,1)$ in $\mathbb{R}^{d}$ and a solution in $C^{2}(B)$ of $\Delta u+g(|x|)+\lambda f(|x|, u)=0$, $u \geq 0$ in $B, u_{\mid \partial B}=0$. If $g(r)=2 d$ and $f(r, u)=r^{2}-1+u, u=1-|x|^{2}+\epsilon w>0$ for $\epsilon>0$ small enough is a non-radial strictly positive solution provided $\lambda$ is an eigenvalue of the operator $(-\Delta)$ in $H_{0}^{1}(B)$ bigger than $\lambda_{2}$. However, if $\lambda$ is small enough, the symmetry results of the B. Gidas, W.-M. Ni et L. Nirenberg theorem in [7] still hold.

PROPOSITION - Let $g$ be a continuous function on $B$ and $f$ a function which is globally Lipschitz in $u$ uniformly in $r$. Then there exists a constant $\Lambda_{2}>0$ such that for any $\left.\lambda \in\right]-\Lambda_{2}, \Lambda_{2}[$, if $u$ is a solution, then it is radially symmetric. If moreover $f$ is increasing in $u$ and if the solution $v$ of $\Delta v+g(|x|)+f(|x|, 0)$ in $B, v_{\mid \partial B}=0$ is nonnegative, then there exists a constant $\left.\Lambda_{1} \in\right] 0, \Lambda_{2}[$ such that for any $\lambda \in] 0, \Lambda_{1}[, u-\lambda v$ is positive and strictly decreasing along any radius of $B$.

When the nonlinearity $f$ is continuous but non-Lipschitz, it is possible to build non-radial positive solutions of $\Delta u+f(u)=0, u \geq 0$ dans $B, u_{\mid \partial B}=0$. In that case, the appropriate notion of symmetry is a notion of local radial symmetry. A solution $u$ is said to be locally radially symmetric if and only if there exists a set of balls $\left(B_{i}\right)_{i=1,2, \ldots N}, B_{i} \subset B=B(0,1)$ such that $u$ is constant on $\partial B_{i}$ and the functions $u_{i}$ defined by $u^{0}=u, u^{i+1}=u^{i} \chi_{B \backslash B_{i}}+u_{\mid \partial B_{i}}^{i} \chi_{B_{i}}$ are still solutions, $u^{i}-u^{i+1}$ is radially symmetric on the ball $B_{i}$ and $u_{N}$ is radially symmetric. This notion of symmetry can easily be generalized to the case where the set of the balls $B_{i}$ is infinite 


\section{J. Dolbeault, P Felmer}

but enumerable, for a nonlinearity which is continuous but not very smooth and has an infinite number of zeros. In the following, we shall call a radially symmetric core any ball on which the solution is radially symmetric and monotone.

Proposition. - Consider a continuous function $f$ on $\mathbb{R}^{+}$and a locally radially symmetric solution $u$ of $\Delta u+f(u)=0, u \geq 0$ dans $B, u_{\mid \partial B}=0$. If $d \geq 2$, then with the above notations the functions $u^{i}-u^{i+1}$ and $u_{N}$ are radially symmetric (up to a translation), positive and decreasing.

Let $D$ be a smooth bounded domain in $\mathbb{R}^{d}$ and assume that $D$ is $x_{1}$-convex, symmetric with respect to the hyperplane $T=\left\{\left(x_{1}, x^{\prime}\right) \in \mathbb{R} \times \mathbb{R}^{d-1}: x_{1}=0\right\}$ and such that for any $\epsilon>0$, there exists a $\delta>0$ for which

$$
\forall \nu \in S^{d-1}, \forall \lambda>\epsilon \text {, if }\left|\nu-e_{1}\right|<\delta \text {, then } D_{\lambda}^{\nu}=\{x-(2 \lambda-x \cdot \nu) \nu: x \in D, x \cdot \nu>\lambda\} \subset D
$$

THEOREM 1. - Let $f: \mathbb{R}^{+} \rightarrow \mathbb{R}$ be a continuous function having the following property: for any $u_{0} \geq 0$, there exists a $\eta>0$ such that on theinterval $] u_{0}-\eta, u_{0}+\eta\left[\cap \mathbb{R}^{+}, f\right.$ is either strictly decreasing or the sum of a Lipschitz and of an increasing function. If $D$ is a smooth bounded domain in $\mathbb{R}^{d}$ satisfying Condition (*), then any solution in $C^{2}(D) \cap C^{1}(\bar{D})$ of $\Delta u+f(u)=0$, $u \geq 0$ in $D, u_{\mid \partial D}=0$ is the sum of a symmetric with respect to $T$ function, decreasing with respect to $x_{1} \geq 0$ and of a locally radially symmetric function whose support is contained in $\left\{x \in D: u_{s}(x)=\left\|u_{s}\right\|_{L^{\infty}(D)}\right\}$, which is a solution of $\Delta u+f\left(u+\left\|u_{s}\right\|_{L^{\infty}(D)}\right)=0$ and has at most a finite number of radially symmetric cores.

Analogous results also hold for an unbounded domain for which there exists an entering cone of directions attached to any point of the boundary: any solution is monotonous with respect to these directions up to locally radially symmetric functions. The method is valid for fully nonlinear elliptic operators as well (see [6] for a proof and more detailed results).

The method is based on the generalization of the argument due to C. Cortázar, M. Elgueta \& P. Felmer in [4] to any of the level sets of $u$ corresponding to values for which $f$ is strictly decreasing (no regularity is required on the boundary), which allows - using a sufficient number of directions - to build a radially symmetric core. Then the generalization of a trick introduced by L.A. Peletier and J. Serrin in [9] and a unique continuation method provides the proof of Theorem 1.

A careful analysis of the radial solutions gives a sufficient condition for locally radially symmetric solutions to be (globally) radially symmetric in the case of a ball, using a continuous Steiner symmetrization developed by F. Brock in [3]. The moving hyperplane method gives (in dimension $d=2$ only) an alternative method which can be generalized to unbounded domains (providing monotonicity results) as well as to fully nonlinear elliptic operators. In this spirit, we may state the following result (see [5] for more details).

THEOREM 2. - Let $f \in C^{0}\left(\mathbb{R}^{+}\right)$be such that either $f(0)>0$ or $\liminf _{u \rightarrow 0_{+}} f(u) / u>-\infty$, and such that for any $\bar{u} \in] 0,+\infty\left[\right.$, if $f(\bar{u})=0, \liminf _{u, v \rightarrow \bar{u}, u \neq v}(f(v)-f(u)) /(v-u)>-\infty$. Then any solution $u$ of of $\Delta u+f(u)=0, u \geq 0$ dans $D, u_{\mid \partial D}=0$ in $C^{1}(\bar{D}) \cap C^{2}(D)$ is symmetric with respect to $T$ if $D$ is a smooth bounded domain in $\mathbb{R}^{2}$ such that Condition (*) is satisfied, and $\frac{\partial u}{\partial x_{1}}\left(x_{1}, x^{\prime}\right)<0$ for any $x_{1}>0$.

The strict decay property is also true for any $x \in D$ and for any direction $\gamma$ close to $e_{1}$ if $x \cdot \gamma>\sup \{y \cdot \gamma: y \in \partial D, \nu(y) \cdot \gamma=0\}$ where $\nu(y)$ is the outgoing normal at $y \in \partial D$. The local inversion argument of Theorem 1, Hopf's lemma and the Maximum Principle show that if $\bar{\lambda}=\sup \left\{x_{1}: \exists x^{\prime} \in \mathbb{R}\right.$ such that $\left(x_{1}, x^{\prime}\right) \in D$ and $\left.\nabla u\left(x_{1}, x^{\prime}\right)=0\right\}$, then $\frac{\partial u}{\partial x_{1}}\left(x_{1}, x^{\prime}\right)<0$, as soon as $x_{1}>\bar{\lambda}$. At $\bar{x}=\left(\bar{\lambda}, \bar{x}^{\prime}\right) \in D$ such that $\frac{\partial u}{\partial x_{1}}(\bar{x})=0, \nabla u(\bar{x})=0$, a blow-up method shows that $\frac{\partial^{2} u}{\partial x_{1}^{2}}(\bar{x})=0$, and since $d=2$, we get $0=-\Delta u(\bar{x})=f(u(\bar{x}))$, which gives the conclusion. 


\section{Introduction}

Le théorème de B. Gidas, W.-M. Ni et L. Nirenberg (voir [7]) affirme que toute solution strictement positive de classe $C^{2}$ de $\Delta u+f(|x|, u)=0, u \geq 0$ dans $B, u_{\mid \partial B}=0$, où $B$ est la boule unité de $\mathbb{R}^{d}$, est à symétrie radiale, c'est à dire ne dépend que de $|x|$, et strictement décroissante en $|x|$ sur l'intervalle ]0,1[, dès lors que $f$ est une fonction décroissante en $|x|$, et somme d'une fonction Lipschitz et d'une fonction croissante en $u$.

Le but de cette note est d'illustrer le rôle des différentes hypothèses de ce théorème et d'annoncer plusieurs résultats nouveaux. Sauf dans la dernière partie, nous traîterons de résultats de symétrie dans la boule.

Exemple 1. Si $w \in C^{2}(B)$ est une fonction propre donné par $\Delta w+\lambda w=0$, correspondant à un $\lambda$ plus grand que la seconde valeur propre $\lambda_{2}$, alors, avec $g(r)=2 d$ et $f(r, u)=r^{2}-1+u$, $u=1-|x|^{2}+\epsilon w>0$ pour $\epsilon>0$ assez petit est une solution non radiale de

$$
\Delta u+g(|x|)+\lambda f(|x|, u)=0, \quad u \geq 0 \text { dans } B, \quad u_{\mid \partial B}=0 .
$$

Exemple 2. Soit $\epsilon>0$, petit, et $u$ une fonction de classe $C^{3}$, positive, telle que

(i) $u(0)=u_{0}>0, u^{\prime}(0)=0, u^{\prime}(r)<0$ pour $r$ compris entre 0 et $\epsilon, u(\epsilon)=a$ et $u^{\prime}(\epsilon)=0$

(ii) $u(r) \equiv a$ pour $r$ compris entre $\epsilon$ et $1-\epsilon$

(iii) $u^{\prime}(r)<0$ pour $\left.\left.r \in\right] 1-\epsilon, 1\right], u(1)=0$.

Pour tout $v \in\left[0, u_{0}\right]$, on peut donc définir $f(v)=-u^{\prime \prime}(r)-\frac{d-1}{r} u^{\prime}(r), r$ étant choisi tel que $u(r)=v$ :

$$
f \in C^{0}\left(\left[0, u_{0}\right]\right) \cap C^{1}(] 0, a[\cup] a, u_{0}[), f(a)=0 \text { et } \lim _{u \rightarrow a} f^{\prime}(u)=-\infty,
$$

et $u$ est solution de

$$
\Delta u+f(u)=0, \quad u \geq 0 \mathrm{dans} B, \quad u_{\mid \partial B}=0 .
$$

Cette équation étant invariante par translation, $\tilde{u}=\left(1-\sum_{i=1}^{N} \chi_{B\left(c_{i}, \epsilon\right)}\right) u+\sum_{i=1}^{N} \chi_{B\left(c_{i}, \epsilon\right)} u\left(.-c_{i}\right)$ est encore une solution, non radiale, strictement positive, de classe $C^{3}$ de $(3)$ dès lors que pour tout $i=1,2, \ldots N, c_{i}$ appartient à $B(0,1)$ et $\epsilon>0$ est choisi suffisamment petit pour que les boules $B\left(c_{i}, \epsilon\right)$ soient contenues dans $B(0,1)$ et deux à deux disjointes.

Réciproquement, on montre que (voir [6]) si $f$ vérifie la propriété (2), alors pour toute solution $u$ de classe $C^{1}$, il existe un nombre fini de boules $B\left(c_{i}, \epsilon_{i}\right)$ telles que $u_{\mid \partial B\left(c_{i}, \epsilon_{i}\right)}=a, \nabla u_{\mid \partial B\left(c_{i}, \epsilon_{i}\right)}=0$, $\operatorname{car} \epsilon_{i} \geq\left(\inf \{u>a: f(u) \geq 0\} \cdot\|\nabla u\|_{L^{\infty}\left(B\left(c_{i}, \epsilon_{i}\right)\right)}^{-1}\right.$. De plus, si $u_{\mid B\left(c_{i}, \epsilon_{i}\right)}$ est radiale, alors $u$ est de classe $C^{2}$ sur $B\left(c_{i}, \epsilon_{i}\right)$.

Cet exemple peut être généralisé dès lors que $f$ admet plusieurs zéros ayant la propriété $(2)$. On peut ainsi construire des solutions non radiales ayant pour niveau critiques de tels zéros de $f$, mais possédant encore une symétrie radiale locale (voir [3]), au sens où il existe un ensemble de boules $\left(B_{i}\right)_{i=1,2, \ldots N}, B_{i} \subset B=B(0,1)$ telles que $u$ est constante sur $\partial B_{i}$ et les fonctions $u_{i}$ définies par $u^{0}=u, u^{i}=u^{i-1} \chi_{B \backslash B_{i}}+u_{\mid \partial B_{i}}^{i-1} \chi_{B_{i}}$ sont encore solutions de (3), $u^{i-1}-u^{i}$ est radiale sur la boule $B_{i}$ et $u_{N}$ est radiale. La notion de solution localement radiale se généralise facilement au cas où l'ensemble des boules $B_{i}$ est infini, dénombrable, pour une nonlinéarité $f$ continue, peu régulière, et possédant un nombre infini de zéros. Dans la suite, nous appelerons cour de symétrie radiale toute boule sur laquelle la solution est radiale et monotone (au sens large).

\section{Positivité, monotonie et solutions localement à symétrie radiale}

Proposition 1. - Soit $f$ une fonction continue sur $\mathbb{R}^{+}$, et $u \geq 0$ une solution localement $\grave{a}$ symétrie radiale de (3) à $N$ fini, avec les notations de l'introduction. Si $d \geq 2$, alors les fonctions $u^{i}-u^{i+1}(i=0,1,2, \ldots N-1)$ et $u_{N}$ sont positives, radiales (à translation près) et décroissantes. 


\section{J. Dolbeault, P Felmer}

En d'autres termes, si $u$ est globalement positive ou nulle, alors sur un cour de symétrie radiale, $u$ est nécessairement décroissante (ce qui entraîne en particulier: $u^{i}-u^{i+1} \geq 0$ ). L'idée principale réside dans le calcul suivant. Considérons $u^{+}$et $u^{-}$des solutions de $\frac{d^{2} u}{d r^{2}}+\frac{N-1}{r} \cdot \frac{d u^{\prime}}{d r}+f(u(r))=0$, définies respectivement sur les intervalles $\left.I^{-}=\right] r_{1}^{-}, r_{0}^{-}$[ et $\left.I^{+}=\right] r_{0}^{+}, r_{1}^{+}\left[\left(\right.\right.$avec $0<r_{1}^{-}<r_{0}^{-} \leq$ $\left.r_{0}^{+}<r_{1}^{+}\right)$, telles que $u\left(r_{0}^{ \pm}\right)=a, \frac{d u^{ \pm}}{d r}\left(r_{0}^{ \pm}\right)=0, u^{ \pm}(r)<a$ où $a>0$ est bien entendu choisi tel que $f(a)=0$. $u^{-}$est croissante sur $I^{-}$et $u^{+}$décroissante sur $I^{+}$, au moins aussi longtemps que $\frac{d u^{ \pm}}{d r}$ ne s'annulle pas. Conformément à la méthode de L.A. Peletier et J. Serrin dans [9], il est possible d'étendre ces solutions de manière unique tant que $\frac{d u^{ \pm}}{d r} \neq 0$. Quitte à diminuer $r_{1}^{-}$et à augmenter $r_{1}^{+}$, nous pouvons supposer que $I^{-}$et $I^{+}$sont les intervalles maximaux dans $\mathbb{R}^{+}$pour lesquels cette propriété est vérifiée. Alors pour tout $r \in I^{-}, \frac{d u^{-}}{d r}(r)=0$ est impossible à moins que $u^{-}(r)<\inf _{s \in I^{+}} u^{+}(s)$. En effet, les fonctions $r^{ \pm}(t)$ telles que $t=u^{ \pm}\left(r^{ \pm}(t)\right)$ sont solutions de $\frac{\left(r^{ \pm}\right)^{\prime \prime}}{\left(\left(r^{ \pm}\right)^{\prime}\right)^{3}}=f(t)+(d-1) \frac{1}{r^{ \pm}\left(r^{ \pm}\right)^{\prime}}$. En multipliant par $\left(r^{ \pm}\right)^{\prime}(t)$ et en intégrant de $u^{+}(r)$ à $a$, on obtient effectivement pour tout $r \in I^{+}$

$$
\begin{aligned}
0 \leq \frac{1}{2}\left(\frac{d u^{+}}{d r}(r)\right)^{2}= & \int_{u^{+}(r)}^{a} f(s) d s+(d-1) \int_{u^{+}(r)}^{a} \frac{d s}{r^{+}(s)\left(r^{+}\right)^{\prime}(s)} \\
& <\int_{u^{+}(r)}^{a} f(s) d s+(d-1) \int_{u^{+}(r)}^{a} \frac{d s}{r^{-}(s)\left(r^{-}\right)^{\prime}(s)}=\frac{1}{2}\left(\frac{d u^{-}}{d r}\left(r^{-}\left(u^{+}(r)\right)\right)\right)^{2} .
\end{aligned}
$$

Le calcul ci-dessus est encore valide si $d u^{-} / d r\left(r_{0}^{-}\right)>0, d u^{+} / d r\left(r_{0}^{+}\right)=0$ et l'on étend sans diffficulté le raisonnement au cas où $d u^{+} / d r$ s'annule sur $I^{+}$en définissant $r^{+}$par $r^{+}(t)=\inf \left\{s>r_{0}^{+}\right.$: $\left.u^{+}(s)=t\right\}$. La Proposition 1 se démontre alors par contradiction en utilisant la définition de la symétrie radiale locale.

\section{Cas où la dépendance dans la variable d'espace n'est pas décroissante}

Proposition 2. - Soit $g$ une fonction continue sur $B$ et $f$ une fonction globalement Lipschitz en $u$ uniformément en $r$. Alors il existe une constante $\Lambda_{2}>0$ telle que pour tout $\left.\lambda \in\right]-\Lambda_{2}, \Lambda_{2}[$, si $u$ est une solution de (1), alors $u$ est radiale. Si de plus $f$ est croissante en $u$ et si la solution $v$ de classe $C^{2}$ de $\Delta v+g(|x|)+f(|x|, 0)$ dans $B, v_{\mid \partial B}=0$, est positive ou nulle, alors il existe une constante $\left.\Lambda_{1} \in\right] 0, \Lambda_{2}$ [ telle que pour tout $\left.\lambda \in\right] 0, \Lambda_{1}[$, si u est une solution de (1), alors $u-\lambda v$ est strictement positive, décroissante le long d'un rayon de $B$.

Remarquons que le résultat de symétrie est indépendant du signe de $u$, ce qui est cohérent avec l'Exemple 1: la positivité et l'unicité sont des propriétés de première valeur propre, la symétrie étant liée à la seconde valeur propre. La première partie de la Proposition 2 est essentiellement connue (voir [1], [8], [10]). La preuve en est assez simple et s'inspire de méthodes de comparaison pour des branches de solutions (voir par exemple [2]). Pour tout $\left(x_{1}, x^{\prime}\right) \in \mathbb{R} \times \mathbb{R}^{d-1} \cap B$, on considère $\bar{u}\left(x_{1}, x^{\prime}\right)=u\left(-x_{1}, x^{\prime}\right)$ et $w=\bar{u}-u$ qui est solution de

$$
\Delta w+\lambda g_{\lambda}(x) w=0 \text { dans } B, \quad w_{\mid \partial B}=0,
$$

où $g_{\lambda}(x)=(f(|x|, \bar{u})-f(|x|, u)) /(\bar{u}-u)$ si $\bar{u}(x) \neq u(x), g_{\lambda}(x)=0$ sinon. On note $\left(g_{\lambda}\right)_{+}=$ $\max \left(0, g_{\lambda}\right)$ et $\left(g_{\lambda}\right)_{-}=\min \left(0, g_{\lambda}\right)$. Soit $\lambda_{ \pm 1}(\lambda)$ la première valeur propre positive (resp. négative) de l'opérateur $-\Delta$ dans l'espace à poids $g_{\lambda}: \lambda_{ \pm 1}(\lambda)= \pm \inf \int_{B}|\nabla v|^{2} d x$ où l'infimum est pris sur l'ensemble des fonctions $v$ de $H_{0}^{1}(B)$ telles que $\int_{B}\left(g_{\lambda}\right)_{ \pm}|v|^{2} d x= \pm 1$, et $\lambda_{ \pm 2}(\lambda)$ la deuxième valeur propre (avec la convention $\lambda_{+i}=+\infty$ si $g_{\lambda}$ est négative, $\lambda_{-i}=-\infty$ si $g_{\lambda}$ est positive). Il est clair que $\left|\lambda_{ \pm 2}(\lambda)\right|>\left|\lambda_{ \pm 1}(\lambda)\right| \geq \lambda_{1}(-\Delta) / K=\Lambda^{*}$, où $\lambda_{1}(-\Delta)$ est la première valeur propre 
du Laplacien dans la boule (avec poids 1 ) et $K$ la constante de Lipschitz de $f$. On peut ainsi définir $\Lambda_{2}=\inf _{\lambda \in \mathbb{R}}\left|\lambda_{ \pm 2}(\lambda)\right| \geq \Lambda^{*}>0$, et donc, si $\lambda \in\left(-\Lambda_{2}, \Lambda_{2}\right)$, les seules solutions non triviales de (4) sont celles qui correspondent à $\lambda=\lambda_{ \pm 1}(\lambda)$. De telles solutions ne changent pas de signe, ce qui est en contradiction avec le fait que $w\left(x_{1}, x^{\prime}\right)=-w\left(-x_{1}, x^{\prime}\right)$ pour tout $\left(x_{1}, x^{\prime}\right) \in B$. Par conséquent, $u$ est symétrique par rapport à l'hyperplan $T=\left\{\left(x_{1}, x^{\prime}\right) \in \mathbb{R} \times \mathbb{R}^{d-1}: x_{1}=0\right\}$, et plus généralement par rapport à tout hyperplan passant par l'origine: $u$ est à symétrie radiale.

Considérons maintenant $w=u-\lambda v$, qui est solution de $\Delta w+\lambda h_{\lambda} w+k_{\lambda}=0$, où $h_{\lambda}(x)=$ $(f(|x|, u)-f(|x|, \lambda v)) /(u-\lambda v)$ si $u(x) \neq \lambda v(x), h_{\lambda}(x)=0$ sinon, et $k_{\lambda}(x)=\lambda[f(|x|, \lambda v(x))-$ $f(|x|, 0)] \geq 0$. De même que ci-dessus, on peut considérer $\mu_{1}(\lambda)$ la première valeur propre positive de l'opérateur $-\Delta$ dans l'espace à poids $h_{\lambda}$. D'une part, on a l'estimation $\Lambda_{1}=\inf _{\lambda \in \mathbb{R}} \mu_{1}(\lambda) \geq$ $\Lambda^{*}>0$, et d'autre part, pour $\left.\lambda \in\right] 0, \Lambda_{1}\left[,-\Delta-\lambda h_{\lambda}\right.$ obéit à un principe du maximum, ce qui montre que $w$ est positive ou nulle. Quitte à diminuer $\Lambda_{1}$, on peut supposer que $\Lambda_{1} \leq \Lambda_{2}, u$ et $v$ sont alors radiales, $-\frac{d^{2}}{d r^{2}}(u-\lambda v)(0)=\lambda k_{\lambda}>0$ (dès lors que $f$ est non constante en $u$ ), ce qui démontre que $\frac{d}{d r}(u-\lambda v)$ est strictement négatif dans un voisinage de $r=0_{+}$, et le lemme de Hopf permet de conclure que $(u-\lambda v)$ est strictement décroissante sur l'intervalle ]0,1]. Remarquons pour finir que si $|\lambda| \leq \Lambda_{1}$, la solution est unique (voir [2]).

\section{Cas où la nonlinéarité n'est pas régulière}

Soit $D$ un domaine borné, régulier de $\mathbb{R}^{d}$ et supposons que $D$ est $x_{1}$-convexe, symétrique par rapport à l'hyperplan $T=\left\{\left(x_{1}, x^{\prime}\right) \in \mathbb{R} \times \mathbb{R}^{d-1}: x_{1}=0\right\}$ et tel que pour tout $\epsilon>0$, il existe un $\delta>0$ pour lequel

$$
\forall \nu \in S^{d-1}, \forall \lambda>\epsilon, \text { si }\left|\nu-e_{1}\right|<\delta, \text { alors } D_{\lambda}^{\nu}=\{x-(2 \lambda-x \cdot \nu) \nu: x \in D, x \cdot \nu>\lambda\} \subset D .
$$

THÉORÈme 1. - Soit $f: \mathbb{R}^{+} \rightarrow \mathbb{R}$ une fonction continue ayant la propriété suivante: pour tout $u_{0} \geq 0$, il existe un réel $\eta>0$ tel que sur l'intervalle $] u_{0}-\eta, u_{0}+\eta\left[\cap \mathbb{R}^{+}\right.$, $f$ est soit strictement décroissante, soit somme d'une fonction Lipschitz et d'une fonction croissante. Si D est un domaine borné de $\mathbb{R}^{d}$ de bord de classe $C^{1}$ vérifiant la condition (5), alors toute solution de classe $C^{2}(D) \cap C^{1}(\bar{D})$ de $\Delta u+f(u)=0, u \geq 0$ dans $D, u_{\mid \partial D}=0$ est la somme d'une fonction symétrique $u_{s}$ par rapport à $T$, décroissante au sens large en $x_{1} \geq 0$ (ainsi que, dans le domaine $D_{\lambda}^{\nu}$, dans toute direction $\nu$ pour laquelle la condition (5) est vérifiée) et d'une fonction localement à symétrie radiale, dont le support est contenu dans $\left\{x \in D: u_{s}(x)=\left\|u_{s}\right\|_{L^{\infty}(D)}\right\}$, qui est solution de $\Delta u+f\left(u+\left\|u_{s}\right\|_{L^{\infty}(D)}\right)=0$ et possède au plus un nombre fini de cœurs à symétrie radiale.

Un résultat analogue peut être énoncé dans le cas d'un domaine non borné pour lequel il existe un cône de directions entrantes en tout point du bord: on montre alors la monotonie de la solution dans ces directions, à des fonctions localement à symétrie radiale près (voir [6]).

La décroissance stricte de $f$ a permis à C. Cortázar, M. Elgueta \& P. Felmer dans [4] de traîter le cas d'une nonlinéarité non Lipschitz en $u=0$. Nous étendons cette approche à tout ensemble de niveau de $u$ correspondant à une valeur pour laquelle $f$ est strictement décroissante (aucune régularité n'est requise au bord) et construisons ainsi une suite finie de sous-domaines à l'aide d'un méthode locale d'hyperplans mobiles. En prenant un nombre suffisant de directions, on montre l'existence d'un cœur à symétrie radiale, puis en généralisant un argument de L.A. Peletier et J. Serrin donné dans [9] et en utilisant une méthode de continuation unique, on montre le Théorème 1. La méthode peut aussi être utilisée dans le cadre d'opérateurs plus généraux, y compris complètement non linéaires.

Une analyse fine des solutions radiales montre que si $\nabla u$ s'annulle, alors il existe un réel $\bar{u}>0$ et deux suites $\left(u_{n}^{-}\right)_{n \in \mathbb{N}}$ et $\left(u_{n}^{+}\right)_{n \in \mathbb{N}}$ telles que $u_{n}^{-}<\bar{u}<u_{n}^{+}$pour tout $n \in \mathbb{N}, \lim _{n \rightarrow+\infty} u_{n}^{ \pm}=\bar{u}$, $f(\bar{u})=0$ et $\left.\lim _{n \rightarrow+\infty} f\left(u_{n}^{ \pm}\right)\right) /\left(u_{n}^{ \pm}-\bar{u}\right)=-\infty$. A l'aide d'une méthode de symétrisation de Steiner 


\section{J. Dolbeault, P Felmer}

continue, F. Brock a récemment démontré - entre autres résultats - dans [3] qu'à l'inverse, si cette condition était violée en tout zéro de $f, u$ était strictement monotone en $x_{1}>0$. Dans le cas d'une boule, il a de plus prouvé qu'une solution positive correspondant à une nonlinéarité continue était localement à symétrie radiale (avec au plus un nombre dénombrable de cœurs à symétrie radiale). La méthode des hyperplans mobiles fournit, en dimension $d=2$ seulement, une approche alternative qui peut être généralisée aussi bien à des opérateurs elliptiques complètement non linéaires qu'à des domaines non bornés (pour lesquels on prouve un résultat de monotonie).

ThÉORÈme 2. - Soit $f \in C^{0}\left(\mathbb{R}^{+}\right)$telle que $f(0)>0$ ou $\liminf _{u \rightarrow 0_{+}} f(u) / u>-\infty$, et telle que pour tout $\bar{u} \in] 0,+\infty\left[\right.$, si $f(\bar{u})=0$, $\liminf _{u, v \rightarrow \bar{u}, u \neq v}(f(v)-f(u)) /(v-u)>-\infty$. Alors toute solution $u$ de $\Delta u+f(u)=0, u \geq 0$ dans $D, u_{\mid \partial D}=0$ dans $C^{1}(\bar{D}) \cap C^{2}(D)$ est symétrique par rapport à $T$ si $D$ est un domaine borné régulier de $\mathbb{R}^{2}$ vérifiant $(5)$, et $\frac{\partial u}{\partial x_{1}}\left(x_{1}, x^{\prime}\right)<0$ dès que $x_{1}>0$.

Bien entendu, la propriété de décroissance stricte reste vraie en $x \in D$ pour toute direction $\gamma$ proche de $e_{1}$ pour autant que $x \cdot \gamma>\sup \{y \cdot \gamma: y \in \partial D, \nu(y) \cdot \gamma=0\}$ où $\nu(y)$ est la normale sortante en $y \in \partial D$, et de manière analogue, on montre des résultats de monotonie pour des domaines non bornés (voir [5]).

L'argument d'inversion locale utilisé dans la preuve du Théorème 1, le lemme de Hopf et le principe du maximum appliqués à l'opérateur obtenu après inversion locale permettent de montrer que pour tout $x_{1}>\bar{\lambda}=\sup \left\{x_{1}: \exists x^{\prime} \in \mathbb{R}\right.$ tel que $\left(x_{1}, x^{\prime}\right) \in D$ et $\left.\nabla u\left(x_{1}, x^{\prime}\right)=0\right\}, \frac{\partial u}{\partial x_{1}}\left(x_{1}, x^{\prime}\right)<0$. En $\bar{x}=\left(\bar{\lambda}, \bar{x}^{\prime}\right) \in D$ tel que $\frac{\partial u}{\partial x_{1}}(\bar{x})=0$, on montre que $\nabla u(\bar{x})=0, \frac{\partial^{2} u}{\partial x_{1}^{2}}(\bar{x})=0$ par un argument de blow-up et, du fait de la dimension $d=2$, on obtient $0=-\Delta u(\bar{x})=f(u(\bar{x}))$, d'où le résultat.

Remerciements. Ce travail a été effectué dans le cadre du contrat ECOS-CONYCIT no. C95E02.

\section{Références bibliographiques}

[1] P. Aviles, Symmetry theorem related to Pompeiu's problem, Am. J. of Math. 108 (1986) 1023-1036.

[2] H. Beresticky, Le nombre de solutions de certains problèmes semi-linéaires elliptiques, J. F. Anal. 40 (1981) 1-29.

[3] F. Brock, Continuous rearrangement and symmetry of solutions of elliptic problems, prétirage.

[4] C. Cortázar, M. Elgueta \& P. Felmer, Symmetry in an elliptic problem and the blow-up set of a quasi-linear heat equation, Comm. in P.D.E. 21 no. 3 \& 4 (1996) 1507-1520.

[5] J. Dolbeault, P. Felmer, Symmetry and monotonicity properties for positive solutions of semi-linear elliptic PDE's, prétirage Ceremade no. 9904 (1999).

[6] J. Dolbeault, P. Felmer, Monotonicity up to radialy symmetric cores of positive solutions to nonlinear elliptic equations: local moving plane techniques and unique continuation in non-Lipschitz case, en préparation.

[7] B. Gidas, W.-M. Ni \& L. Nirenberg, Symmetry and related properties via the maximum principle, Comm. Math. Phys. 68 (1979) 209-243.

[8] A.C. Lazer \& P.J. McKenna, A symmetry theorem and applications to nonlinear partial differential equations, J. of Diff. Eq. 72 (1988) 95-106.

[9] L. A. Peletier \& J. Serrin, Uniqueness of nonnegative solutions of semilinear equations in $\mathbb{R}^{N}$, J. Diff. Eq. Vol. 61 (1986) 380-397.

[10] M. Willem, Bifurcation, symmetry and Morse theory, Boll. U.M.I. (7) 3-B (1989) 17-27. 